\title{
Association between thrombocytopenia and platelet profile with morbidity/mortality of severe and non-severe COVID-19 patients
}

\author{
Laya Jalilian Khave ${ }^{1}$ (1), Parisa Zafari² (1), Ali Pirsalehi ${ }^{10}$, Sina Salari3* (1), \\ Ahmadreza Baghestani4 (10, Mohammad Esmaeil Akbari ${ }^{5}$ (D), Davood Bashash ${ }^{6 *}$
}

\section{SUMMARY}

OBJECTIVE: Coronavirus disease 2019 (COVID-19) is a novel infectious viral disease that can be associated with changes in platelet counts. Thrombocytopenia is a risk factor for increased mortality and morbidity among these patients. In this study, we aimed to measure the platelet count of COVID-19 patients and find the association with morbidity and mortality after following up.

METHODS: This study was conducted on 1,320 confirmed COVID-19 patients who were admitted to the Ayatollah Taleghani and Shohada Tajrish Hospital in Tehran, Iran. The diagnosis of COVID-19 was confirmed by standard protocols. The data on the platelet profile were retrospectively extracted from patients' electronic medical records consisted of platelet counts on admission, the next 7 days during the hospital stay, and on discharge. Patients were categorized into two groups, namely, "non-severe presentation" and "severe presentation" based on clinical signs.

RESULTS: There was no significant difference in platelet counts and thrombocytopenia between severe and non-severe, survivors and nonsurvivors, and severe survivors and severe non-survivors groups at the time of admission to the hospital. After 7 days, a trend toward an increase in platelet counts was seen in non-severe patients, survivors, and severe compared with severe patients, non-survivors, and severe non-survivors, respectively.

CONCLUSIONS: Thrombocytopenia and thrombotic complications in COVID-19 patients are common and lead to a higher mortality rate. KEYWORDS: COVID-19. Morbidity. Mortality. Platelet. Thrombocytopenia.

\section{INTRODUCTION}

Coronavirus disease 2019 (COVID-19) started with an outbreak in Wuhan, China, in December 2019 and rapidly evolved into a global pandemic within a few months, resulting in 15,099,548 confirmed cases and 619,605 deaths worldwide till date ${ }^{1}$, and the numbers are growing promptly. Although the mortality rates are low compared to other diseases caused by the coronavirus family, the fatality rate of $10 \%$ in severe acute respiratory syndrome (SARS) and 37\% in the Middle East respiratory syndrome (MERS) due to the alarming rates of transmission,

\footnotetext{
'Shahid Beheshti University of Medical Sciences, School of Medicine, Ayatollah Taleghani Hospital, Department of Internal Medicine - Tehran, Iran. ${ }^{2}$ Mazandaran University of Medical Sciences, Faculty of Medicine, Department of Immunology - Sari, Iran.

${ }^{3}$ Shahid Beheshti University of Medical Sciences, Taleghani Hospital, Hematology and Bone Marrow Transplantation, Department of Medical Oncology - Tehran, Iran.

${ }^{4}$ Shahid Beheshti University of Medical Sciences, Faculty of Paramedical Sciences, Physiotherapy Research Center and Department of Biostatistics - Tehran, Iran.

${ }^{5}$ Shahid Beheshti University of Medical Sciences, Cancer Research Center - Tehran, Iran.

${ }^{6}$ Shahid Beheshti University of Medical Sciences, School of Allied Medical Sciences, Department of Hematology and Blood Banking - Tehran, Iran. *Corresponding author: s.salari@sbmu.ac.ir, d.bashash@sbmu.ac.ir

Conflicts of interest: the authors declare there is no conflicts of interest. Funding: Shahid Beheshti University of Medical Science.

Received on July 31, 2021. Accepted on September 02, 2021.
} 
the pandemic is causing a huge burden on countries' resources and economy ${ }^{2}$. The rising mortality and morbidity rates are worrisome particularly in resource-restricted countries with health care systems already facing shortages ${ }^{3}$.

COVID-19 was primarily regarded as respiratory disease, but the emerging data revealed that it should rather be recognized as a systemic illness, involving not only the respiratory system but also multiple organs such as the cardiovascular, neurological, gastrointestinal, hematopoietic, and immune sys$\operatorname{tem}^{4-6}$. Numerous studies have focused on hematological and hemostatic abnormalities - common and significant among severe cases - in COVID-19, highlighting their capacity to predict disease progression and prognosis ${ }^{7-9}$. Among the hematological findings, lymphopenia, neutrophilia, thrombocytopenia, leukocytosis, and/or leukopenia have been observed ${ }^{10-12}$; of those, the platelet count is an available and affordable factor with evident association with COVID-19 severity and possibly mortality ${ }^{13,14}$. Considering the lack of effective therapy and the heavy burden on the health care system, having convenient tools for predicting the prognosis can play a vital role. Thrombocytopenia was previously identified and used as a major prognostic factor in SARS with fair accuracy ${ }^{15,16}$. It can be effectively used in COVID-19 likewise, but to measure the extent, further investigations are in demand and according to the rapidly evolving situation, soon. In this study, we described the epidemiology of thrombocytopenia and platelet profile alterations in a relatively large population of 1,019 confirmed COVID-19 patients and explored the possible link to morbidity and mortality among them.

\section{METHODS}

\section{Participants and procedures}

A total of 1,320 confirmed COVID-19 patients who were admitted to the Ayatollah Taleghani and Shohada Tajrish Hospital in Tehran, Iran, was recruited in a retrospective double-center study from February 20 to May 20, 2020. The diagnosis of COVID-19 was confirmed by:

1. Polymerase chain reaction (PCR) analysis; and

2. Chest computed tomography (CT) inspection. SARS coronavirus 2 (SARS-CoV-2)-contaminated swab specimens from the upper respiratory tract were obtained, maintained in a virus-transport medium, and detected by real-time reverse transcriptase PCR (RT-PCR) analysis.

The amplification arrangements were as followed: $50^{\circ} \mathrm{C}$ for $15 \mathrm{~min}, 95^{\circ} \mathrm{C}$ for $3 \mathrm{~min}, 45$ cycles of $95^{\circ} \mathrm{C}$ for $15 \mathrm{~s}$, and $60^{\circ} \mathrm{C}$ for $30 \mathrm{~s}$. CT scan was requested for all admitted patients with common COVID-19 signs and symptoms such as fever, cough, dyspnea, pleuritic chest pain, decreased $\mathrm{O}_{2}$ saturation, and also abnormal lung auscultation and evaluated by an adaptive radiologist with precise consideration of all imaging features including pure consolidation, pure ground-glass opacity (GGO), mixed GGO and consolidation, reversed halo, intralesional traction bronchiectasis, crazy-paving, intralesional vascular enlargement, linear opacities, pleural effusion, and pericardial effusion. The affected lung lobes were counted, and lesions were categorized as peripheral or central based on lesion location. A thin-section CT involvement was calculated based on the extent of lung involvement. This study was approved by the Ethics Committee of the Shahid Beheshti University of Medical Sciences.

Vital signs of all patients, including blood pressure, respiratory rate, heart rate, and temperature on admission, were measured and demographic baseline information were recorded. The data on the platelet profile were retrospectively extracted from patients' electronic medical records of the Ayatollah Taleghani and Shohada Tajrish Hospital. The profile for each patient consisted of platelet counts on admission, the next 7 days during the hospital stay, and on discharge. For this matter, 301 patients with an incomplete laboratory profile were excluded from this study, and the participants' count with complete baseline and laboratory information dropped to 1,019 cases. Thrombocytopenia was defined as the platelet count under 150,000 platelets per microliter of blood.

Patients were categorized into two groups, namely, "non-severe presentation" and "severe presentation." They were defined as severe if admitted to ICU or died during the course of disease progression or had severe hypoxia $\left(\mathrm{O}_{2}\right.$ saturation $\left.\leq 85 \%\right)$ on admission or had respiratory rate $\geq 30$ on admission and non-severe otherwise. The severe group was also divided into two subgroups of severe survivors and non-survivors.

\section{Statistical analysis}

The continuous variables were examined to determine the normality of the distribution using histograms, measures of skewness and kurtosis, and Kolmogorov-Smirnov test. The normally distributed variables were described as the means \pm standard deviation (SD), and the skewed distributed variables were expressed as the median and interquartile range $(25-75 \%)$. Categorical variables were summarized as frequencies (percentage). The normally distributed continuous variables were compared between non-severe and severe groups using the two independent sample $t$-test and non-normally distributed variables with the Mann-Whitney $U$ test. The comparisons of categorical variables between groups were conducted using the chi-square test of independence. 
All tests were two-sided, and a $\mathrm{p}<0.05$ was considered to indicate a statistically significant difference. All the statistical analyses were performed using the IBM SPSS version 24.0 (IBM Corp., Armonk, NY, USA).

\section{RESULTS}

\section{Baseline characteristics of patients with COVID-19}

A total of 1,320 patients with confirmed COVID-19 diagnosis was enrolled in this study. Patients were categorized into two groups, namely, "non-severe presentation" and "severe presentation" accounting for 74.16 and $25.75 \%$ of total patients. Of note, $13.9 \%$ of patients died during the study while most of them $(85.98 \%)$ survived, and $60.9 \%$ of all patients were males with similar distribution in both non-severe and severe groups (61.69 and 57.9\%). The similar distribution in both groups was also true for the female sex $(38.1 \%$ non-severe and $42.1 \%$ severe groups). Most non-severe participants were at the younger end of the age spectrum, and controversially, most participants in the severe group were older. Notably, $52.8 \%$ of all patients were 50 years old and higher, and while this applied to a significant count of $79.4 \%$ of the severe group in this age range, this highlights the significant effect of age in severe and non-severe groups. In contrast, while men were the most diagnosed patients overall, the role of sex-affecting severity was not significant (Table 1).

\section{The admission and discharge platelet counts and thrombocytopenia}

There was no significant difference in platelet counts and thrombocytopenia between severe and non-severe, survivors and non-survivors, and severe survivors and severe non-survivors groups at the time of admission to the hospital; however, this difference dramatically changed while discharge. Regarding the platelet counts, these alternations were so impressive in the case of survivors and non-survivors as well as severe survivor and severe non-severe groups. Also, severe patients, non-survivors, and severe non-survivors showed higher thrombocytopenia and lower platelet counts compared with non-severe patients, survivors, and severe survivors, respectively, at the time of discharge (Figure 1).

\section{Alteration in the platelet counts and thrombocytopenia during a 7-day follow-up}

The platelet count did not show a significant difference between severe and non-severe, survivors and non-survivors, and severe survivors and severe non-survivors groups on the admission day. After 7 days, a trend toward an increase in platelet counts was seen in non-severe patients, survivors, and severe compared with severe patients, non-survivors, and severe non-survivors,

Table 1. Baseline characteristics of admitted COVID-19 patients.

\begin{tabular}{|c|c|c|c|c|c|c|c|}
\hline Variables & $\begin{array}{c}\text { All patients } \\
\text { n (\%); mean } \\
( \pm S D)\end{array}$ & $\begin{array}{c}\text { Non-severe* } \\
\mathrm{n}(\%) ; \text { mean } \\
( \pm \mathrm{SD})\end{array}$ & $\begin{array}{c}\text { Severe } \\
\mathrm{n}(\%) ; \text { mean } \\
( \pm \mathrm{SD})\end{array}$ & $p$-value & $\begin{array}{c}\text { Survivors } \\
\text { n (\%); mean } \\
( \pm S D)\end{array}$ & $\begin{array}{c}\text { Non-survivors } \\
\mathrm{n}(\%) ; \text { mean } \\
( \pm \mathrm{SD})\end{array}$ & $p$-value \\
\hline Sample size & $1,320(100)$ & $979(74.16)$ & $340(25.75)$ & & $1,135(85.98)$ & $184(13.93)$ & \\
\hline Age (years) & $52.15( \pm 19.22)$ & $47.84( \pm 17.58)$ & $64.59( \pm 18.32)$ & \multirow{6}{*}{0.000} & $49.29( \pm 18.147)$ & $69.82( \pm 15.98)$ & \multirow{6}{*}{0.000} \\
\hline$\leq 39$ & $406(30.8)$ & $371(37.9)$ & $35(10.3)$ & & $401(35.3)$ & $5(2.7)$ & \\
\hline $40-49$ & $216(16.4)$ & $181(18.5)$ & $35(10.3)$ & & 204 (18.0) & $12(6.5)$ & \\
\hline $50-59$ & $201(15.2)$ & $156(15.9)$ & $45(13.2)$ & & $178(15.7)$ & $23(12.5)$ & \\
\hline $60-69$ & $196(14.9)$ & $131(13.4)$ & 65 (19.1) & & $161(14.2)$ & 35 (19.0) & \\
\hline$\geq 70$ & $301(22.7)$ & $140(14.3)$ & $160(47.1)$ & & $191(16.8)$ & $109(59.2)$ & \\
\hline \multicolumn{8}{|l|}{ Sex } \\
\hline Female & $516(39.1)$ & $373(38.1)$ & $143(42.1)$ & \multirow{2}{*}{0.193} & 442 (38.9) & $74(40.2)$ & \multirow{2}{*}{0.736} \\
\hline Male & 804 (60.9) & 607 (61.69) & 197 (57.9) & & $694(61.1)$ & $110(59.8)$ & \\
\hline \multirow{2}{*}{$\begin{array}{l}\text { Platelet } \\
\text { count }\end{array}$} & $\begin{array}{c}\mathrm{n}(\%) \\
\text { median (IQR) }\end{array}$ & $\begin{array}{c}\mathrm{n}(\%) \\
\text { median (IQR) }\end{array}$ & $\begin{array}{c}\mathrm{n}(\%) ; \\
\text { median (IQR) }\end{array}$ & \multirow{2}{*}{0.100} & $\begin{array}{c}\mathrm{n}(\%) \\
\text { median (IQR) }\end{array}$ & $\begin{array}{c}\mathrm{n}(\%) \\
\text { median (IQR) }\end{array}$ & \multirow{2}{*}{0.055} \\
\hline & $\begin{array}{c}176.5 \\
(133.0-246.5)\end{array}$ & $\begin{array}{c}177.0 \\
(139.0-224.0)\end{array}$ & $\begin{array}{c}176.5 \\
(133.0-246.5)\end{array}$ & & $\begin{array}{c}177.0 \\
(139.0-224.0)\end{array}$ & $\begin{array}{c}181.0 \\
(122.0-271.0)\end{array}$ & \\
\hline
\end{tabular}

* Severe patients are defined as cases (i) admitted to intensive care unit or (ii) died during the course of disease progression or (iii) had severe hypoxia $\left(\mathrm{O}_{2}\right.$ saturation $\left.\leq 85 \%\right)$ on admission or (iv) had respiratory rate $\geq 30$ on admission. SD: standard deviation; IQR: interquartile range. 


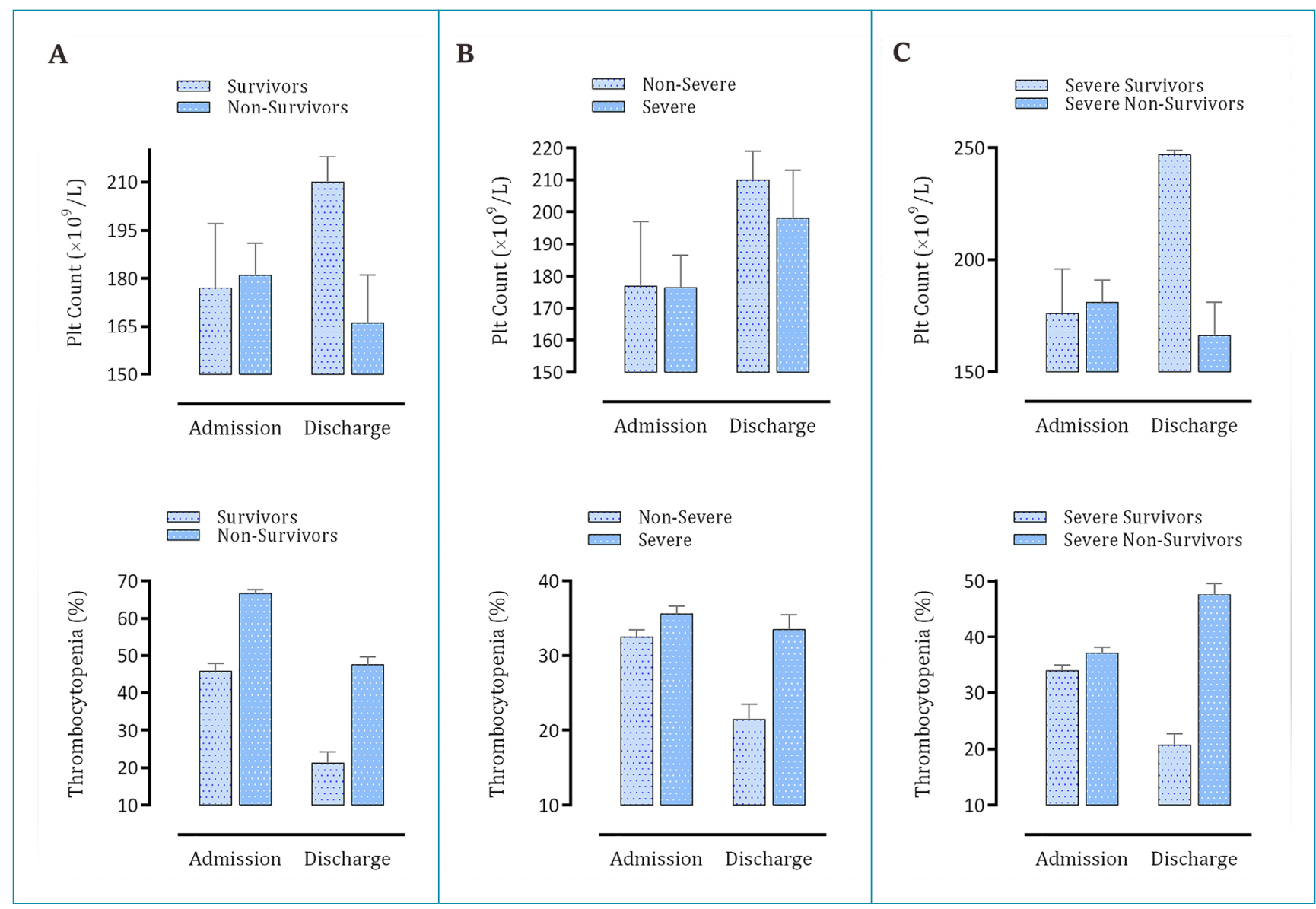

Figure 1. The admission and discharge platelet counts and thrombocytopenia divided by mortality and morbidity.

respectively. Thrombocytopenia proportions did not show the same pattern in all the three groups on the admission day. After following up, the maximum difference of thrombocytopenia occurred between severe survivors and severe non-survivors with a higher magnitude in the non-survivors. Also, thrombocytopenia was higher in severe patients and non-survivors rather than non-severe and survivors after 7 days, respectively (Figure 2).

\section{DISCUSSION}

Mounting evidence has shown that thromboembolic complications resulting from COVID-19 disease are one of the main reasons for sudden deterioration and death ${ }^{17}$. Platelets play a pivotal role in thrombogenesis. An increased platelet activation leading to platelet aggregation, platelet spreading, $\alpha$-granule secretion, and dense granule release is related to thrombosis in COVID-19 $9^{18}$. It has been shown that the incidence of thromboembolic events in severe patients and non-survivors is much higher compared with their non-severe counterparts and survivors $^{1,20}$. This is in harmony with our results showing a significantly decreased platelet count in severe and non-survivor patients rather than non-severe and non-survivor patients, respectively. Although the difference was not significant in admission, it was shown that the platelet count can predict the mortality rate and disease orientation. Also, the platelet count can be considered as an indicator of disease severity. During the disease course, the platelet count showed more change in non-survivors compared with severe and non-severe groups. Although thrombocytopenia is often believed to be an indicator of bleeding, no history of bleeding was reported in COVID-19 patients during hospitalization. Bowles et al. reported that the frequency of bleeding events was 0 in 35 COVID-19 patients who suffered from a prolonged activated partial-thromboplastin time $(\mathrm{aPTT})^{21}$ which may result from the nature of severe hypercoagulative rather than a hypocoagulative state of COVID-19 coagulation pattern. The possible mechanisms by which COVID-19 leads to thrombocytopenia might be that:

1. The suppression of hematopoiesis caused by an impaired hematopoietic microenvironment resulting from systemic inflammation or cytokine storm $^{22}$; 


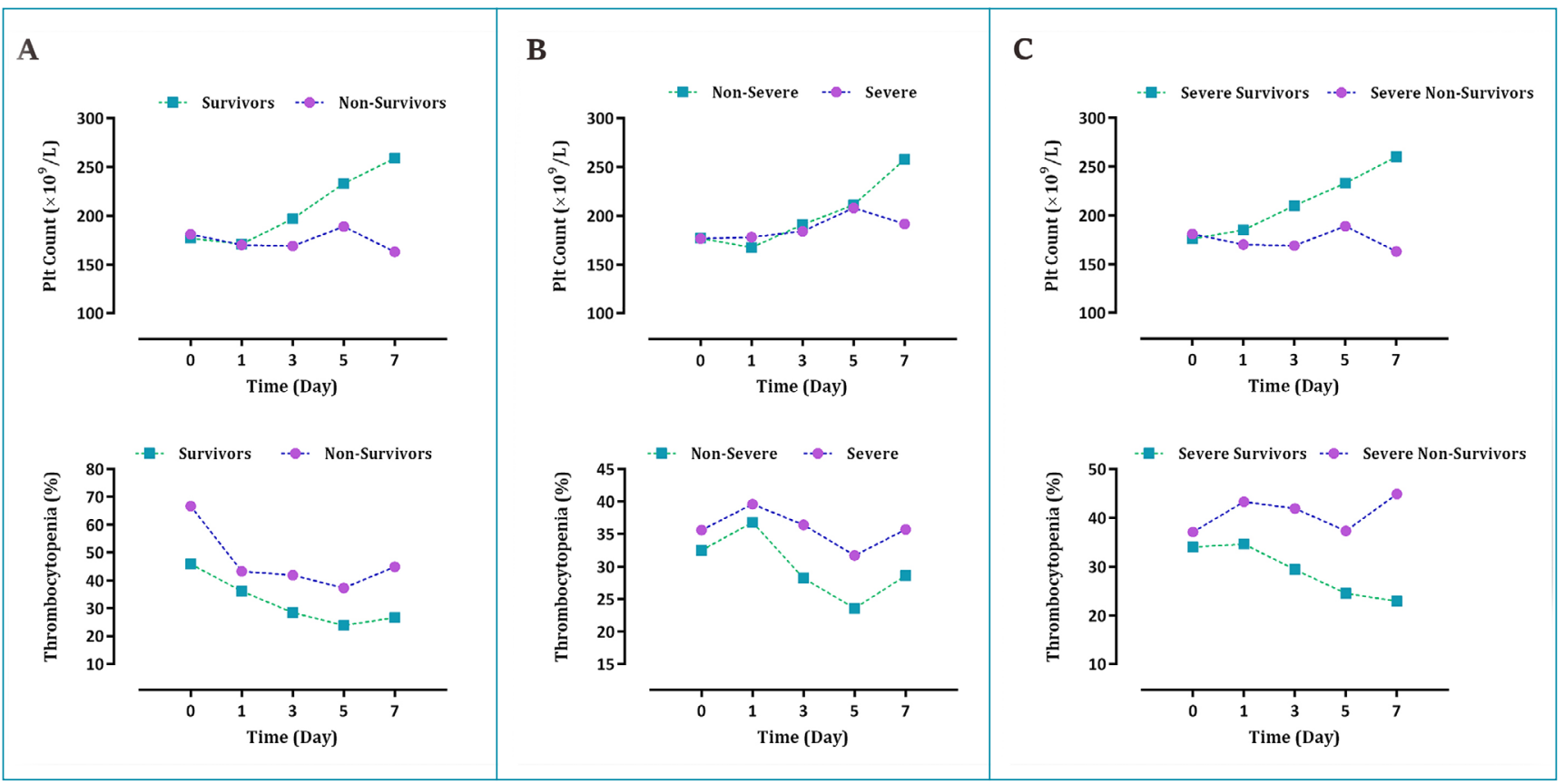

Figure 2. Platelet counts and thrombocytopenia during a 7-day follow-up course divided by mortality and morbidity.

2. The infection of hematopoietic stem cells or megakaryocytes through angiotensin-converting enzyme 2 (ACE2), CD13, or CD66a like other coronavirus infections ${ }^{23}$;

3 . The cross-reaction of antiviral antibodies with hematopoietic cells and platelets like anti-adenovirus antibody reaction with platelet integrin GPIIb/IIIa ${ }^{23}$;

4. The scavenging of activated platelet by splenic/hepatic macrophages; and

5. The increased consumption of platelets caused by thrombotic microangiopathy and disseminated intravascular coagulation $^{24}$. Also, Chen et al. indicated the delayedphase thrombocytopenia resulting from the impaired maturation of megakaryocytes in COVID-19 patients ${ }^{25}$. Following up patients after 7 days revealed that higher thrombocytopenia is related to severe patients and non-survivors rather than non-severe and survivors, respectively. It seems that thrombocytopenia and thrombotic complications in COVID-19 patients are common and lead to a higher mortality rate, but significant bleeding reports are rare among these patients. Taken together, dynamic monitoring, early detection, and thromboprophylaxis can help us to control the platelet count in patients not contraindicated for treatments.

\section{CONCLUSIONS}

Thrombocytopenia and thrombotic complications in COVID-19 patients are common leading to a higher mortality rate.

\section{AUTHORS" CONTRIBUTIONS}

LJK: Investigation, Writing - review \& editing. PZ: Investigation, Writing - review \& editing. AP: Formal analysis. SS: Conceptualization, Data Curation, Investigation, Methodology, Writing - original draft, Writing - review \& editing. AB: Formal analysis. MEA: Investigation, Writing - review \& editing. DB: Conceptualization, Data Curation, Investigation, Methodology, Writing - original draft, Writing - review \& editing.

\section{REFERENCES}

1. Ali SA, Baloch M, Ahmed N, Ali AA, lqbal A. The outbreak of Coronavirus Disease 2019 (COVID-19)-an emerging global health threat. J Infect Public Health. 2020;13(4):644-6. https:// doi.org/10.1016/j.jiph.2020.02.033
2. Petrosillo N, Viceconte G, Ergonul O, Ippolito G, Petersen E. COVID-19, SARS and MERS: are they closely related? Clin Microbiol Infect. 2020;26(6):729-34. https://doi.org/10.1016/j. cmi.2020.03.026 
3. Shadmi E, Chen Y, Dourado I, Faran-Perach I, Furler J, Hangoma P, et al. Health equity and COVID-19: global perspectives. Int J Equity Health. 2020;19(1):104. https://doi.org/10.1186/ s12939-020-01218-z

4. Gavriatopoulou M, Korompoki E, Fotiou D, Ntanasis-Stathopoulos I, Psaltopoulou T, Kastritis E, et al. Organ-specific manifestations of COVID-19 infection. Clin Exp Med. 2020;20(4):493-506. https://doi.org/10.1007/s10238-020-00648-x

5. Zhang Y, Geng X, Tan Y, Li Q, Xu C, Xu J, et al. New understanding of the damage of SARS-CoV-2 infection outside the respiratory system. Biomed Pharmacother. 2020;127:110195. https://doi. org/10.1016/j.biopha.2020.110195

6. Zheng KI, Feng G, Liu WY, Targher G, Byrne CD, Zheng MH. Extrapulmonary complications of COVID-19: a multisystem disease? J Med Virol. 2021;93(1):323-35. https://doi. org/10.1002/jmv.26294

7. Toledo SLO, Nogueira LS, Carvalho MG, Rios DRA, Pinheiro MB. COVID-19: review and hematologic impact. Clin Chim Acta. 2020;510:170-6. https://doi.org/10.1016/j.cca.2020.07.016

8. Rahi MS, Jindal V, Reyes SP, Gunasekaran K, Gupta R, Jaiyesimi I. Hematologic disorders associated with COVID-19: a review. Ann Hematol. 2021;100(2):309-20. https://doi.org/10.1007/ s00277-020-04366-y

9. Vajari MK, Shirin M, Pourbagheri-Sigaroodi A, Akbari ME, Abolghasemi H, Bashash D. COVID-19-related coagulopathy: a review of pathophysiology and pharmaceutical management. Cell Biol Int. 2021;45(9):1832-50. https://doi.org/10.1002/ cbin. 11623

10. Liu $X$, Zhang $R$, He G. Hematological findings in coronavirus disease 2019: indications of progression of disease. Ann Hematol. 2020;99(7):1421-8. https://doi.org/10.1007/s00277020-04103-5

11. Shahri MK, Niazkar HR, Rad F. COVID-19 and hematology findings based on the current evidences: a puzzle with many missing pieces. Int J Lab Hematol. 2021;43(2):160-8. https:// doi.org/10.1111/ijlh.13412

12. Kosmeri C, Koumpis E, Tsabouri S, Siomou E, Makis A. Hematological manifestations of SARS-CoV-2 in children. Pediatr Blood Cancer. 2020;67(12):e28745. https://doi. org/10.1002/pbc.28745

13. Ozcelik N, Ozyurt S, Kara BY, Gumus A, Sahin U. The value of the platelet count and platelet indices in differentiation of COVID-19 and influenza pneumonia. J Med Virol. 2021;93(4):2221-6. https://doi.org/10.1002/jmv.26645

14. Hadid T, Kafri Z, Al-Katib A. Coagulation and anticoagulation in COVID-19. Blood Rev. 2021;47:100761. https://doi. org/10.1016/j.blre.2020.100761

15. Lippi G, Plebani M, Henry BM. Thrombocytopenia is associated with severe coronavirus disease 2019 (COVID-19) infections: a meta-analysis. Clin Chim Acta. 2020;506:145-8. https://doi. org/10.1016/j.cca.2020.03.022

16. Bao C, Tao X, Cui W, Yi B, Pan T, Young KH, et al. SARS-CoV-2 induced thrombocytopenia as an important biomarker significantly correlated with abnormal coagulation function, increased intravascular blood clot risk and mortality in COVID-19 patients. Exp Hematol Oncol. 2020;9:16. https:// doi.org/10.1186/s40164-020-00172-4

17. Bikdeli B, Madhavan MV, Jimenez D, Chuich T, Dreyfus I, Driggin E, et al. COVID-19 and thrombotic or thromboembolic disease: implications for prevention, antithrombotic therapy, and follow-up: JACC state-of-the-art review. J Am Coll Cardiol. 2020;75(23):2950-73. https://doi.org/10.1016/j. jacc.2020.04.031

18. Zhang S, Liu Y, Wang $X$, Yang L, Li H, Wang Y, et al. SARS-CoV-2 binds platelet ACE2 to enhance thrombosis in COVID-19. J Hematol Oncol. 2020;13(1):120. https://doi.org/10.1186/ s13045-020-00954-7

19. Lu L, Zhang $H$, Zhan M, Jiang J, Yin H, Dauphars DJ, et al. Antibody response and therapy in COVID-19 patients: what can be learned for vaccine development? Sci China Life Sci. 2020;63(12):1833-49. https://doi.org/10.1007/s11427-0201859-y

20. Helms J, Tacquard C, Severac F, Leonard-Lorant I, Ohana M, Delabranche $X$, et al. High risk of thrombosis in patients with severe SARS-CoV-2 infection: a multicenter prospective cohort study. Intensive Care Med. 2020;46(6):1089-98. https://doi. org/10.1007/s00134-020-06062-x

21. Bowles L, Platton S, Yartey N, Dave M, Lee K, Hart DP, et al. Lupus anticoagulant and abnormal coagulation tests in patients with Covid-19. N Engl J Med. 2020;383(3):288-90. https:// doi.org/10.1056/NEJMc2013656

22. Valletta $S$, Thomas $A$, Meng $Y$, Ren $X$, Drissen $R$, Sengül $H$, et al. Micro-environmental sensing by bone marrow stroma identifies IL-6 and TGF $\beta 1$ as regulators of hematopoietic ageing. Nat Commun. 2020;11(1):4075. https://doi.org/10.1038/ s41467-020-17942-7

23. Amgalan A, Othman M. Exploring possible mechanisms for COVID-19 induced thrombocytopenia: unanswered questions. J Thromb Haemost. 2020;18(6):1514-6. https://doi.org/10.1111/ jth. 14832

24. Levi M, Thachil J, Iba T, Levy JH. Coagulation abnormalities and thrombosis in patients with COVID-19. Lancet Haematol. 2020;7(6):e438-e40. https://doi.org/10.1016/S23523026(20)30145-9

25. Chen W, Li Z, Yang B, Wang P, Zhou Q, Zhang Z, et al. Delayedphase thrombocytopenia in patients with coronavirus disease 2019 (COVID-19). Br J Haematol. 2020;190(2):179-84. https:// doi.org/10.1111/bjh.16885 